\title{
Los conceptos y su representación: Una perspectiva terminológica para el tratamiento temático de la información
}

\section{Mario Guido Barité Roqueta}

Escuela Universitaria de Bibliotecología y Ciencias Afines

Universidad de la República Oriental del Uruguay

\section{1 . Resumen}

El control de vocabulario y la recuperación de información mediante claves temáticas son las dos expresiones más representativas del trabajo con conceptos en Bibliotecología. Sin embargo, la tendencia en el área ha sido trabajar apenas con las etiquetas o "fachadas" de los conceptos, sin considerar los aspectos relativos a la construcción y definición de los mismos. Desde una visión terminológica se describe y ejemplifica el procedimiento de definición por comprensión, y se identifican cuatro formas de representación conceptual. Se estudia sucintamente la relación dialéctica existente entre el concepto y su representación lingüística, y se da cuenta de posibles aplicaciones metodológicas y teóricas para la expresión de el conocimiento en el área de tratamiento temático de la información (Autor).

Descriptores: Teoria de los conceptos. Terminología. Concepto. Control de vocabulario.

\subsection{Abstract}

Vocabulary control and information retrieval by means of subject keys are the two most representative expressions of the work with concepts in the field of Library and Information Science. However, the main trend in the area has been to work only with the tags of the concepts, without considering aspects related with their construction and definition. From a terminological point of view, the procedure for creating definitions is described and exemplified, and four ways of conceptual representation are identified. The dialectic relation between the concept and its linguistic representation is studied. Finally, the various methodological and theoretical applications of the model for subject representation are considered. 
Keywords: Concept theory. Terminology. Concept. Vocabulary control.

\section{1. introducción}

Resulta ocioso justificar la importancia que tradicionalmente ha tenido el manejo de conceptos para tareas de representación del contenido temático de documentos, en Bibliotecología y Documentación. Son muy variadas las instancias y prácticas profesionales en las que los profesionales del área trabajamos, de un modo u otro, con conceptos, a saber:

- Análisis de contenido y representación de conocimiento (clasificación e indización).

- Elaboración de resúmenes y extracción de terminología de dichos resúmenes para la realización de índices.

- Procesos de almacenamiento y recuperación de información.

- Referencia.

- Construcción y evaluación de lenguajes documentales.

- Diseño de bases de datos.

Estas prácticas pueden sistematizarse, a su vez, dentro de dos grandes tipos de operaciones:

a) Control de vocabulario para el almacenamiento de la información: esto es, la necesidad de formalizar y normalizar los símbolos utilizados para identificar y representar el contenido de los documentos. Como apunta Novellino (1996, p. 38) "la principal característica del proceso de representación de la información es la sustitución de una entidad lingüística extensa y compleja (el texto del documento) por su descripción abreviada", la cual funciona "como un artificio para enfatizar lo que es esencial en el documento". El control de vocabulario comprende, además del esfuerzo de sistematización sobre cada concepto en particular, el establecimiento de las relaciones recíprocas entre los términos y su consecuencia inevitable: la creación de una estructura de conceptos que adquirirá configuraciones particulares (sistemas de clasificación, nomenclaturas, tesauros, listas de encabezamientos, etc.) de acuerdo con la finalidad perseguida. Como manifiestan Cintra y sus colaboradores (1994, p. 31), para lograr el control de vocabulario "los lenguajes documentales incorporan procedimientos para la normalización gramatical y semántica. La normalización gramatical refiere a la forma de presentación de sus elementos en cuanto al género (generalmente masculino), número (uso de singulares y plurales) y grado. La normalización semántica procura garantizar la univocidad en la representación de conceptos y 
nociones de las áreas de especialidad". Dicho de otra manera, mientras la normalización gramatical apunta a formalizar la expresión externa y visible de un concepto (una palabra, una expresión compuesta, un símbolo codificado), la normalización semántica procura establecer una significación propia y precisa para cada término. La normalización semántica es menos aparente en un lenguaje documental porque, salvo cuando se agregan notas de alcance o breves definiciones, esa normalización sólo surge indirectamente de la ubicación relativa del término en una disciplina, y del entramado de relaciones género/especie, todo/parte, asociativas o sinonímicas que le son adjudicadas.

b) Recuperación de información mediante claves temáticas: el control de vocabulario no tiene una justificación por sí mismo; por el contrario, se legitima como constituyente infraestructural para permitir cualquier tipo de almacenamiento y recuperación de información. En ese sentido está relacionado directamente con el procesamiento temático de documentos y su incorporación a un sistema de información, prefigurando y estableciendo las claves de acceso temático para su utilización y reutilización por usuarios indiscriminados. El diseño de formatos de salida y la formulación de estrategias de búsqueda compatibles con ellos mediante procedimientos calificados caen de lleno en este punto.

Más allá de la recurrencia de esas prácticas significativas en nuestra profesión, es justo confesar que manejar conceptos no significa necesariamente dominarlos, en el sentido de conocer su estructura, sus elementos integradores, su funcionalidad, su ciclo vital, las reglas y los fenómenos de orden lingüístico, terminológico y comunicacional que se generan en torno a ellos. Si concebimos a una noción o concepto como un iceberg, de modo tal que lo que queda por debajo de la línea de agua sea la conceptualización misma (la delimitación nocional de algo), y lo que se exhiba por encima de esa línea sea la palabra o el símbolo que expresa ese concepto, costará poco demostrar que los bibliotecólogos hemos prestado atención casi exclusiva a las etiquetas de los conceptos (es decir, a la parte visible del iceberg) en detrimento del análisis de las conceptualizaciones mismas.

En la medida que tradicionalmente hemos puesto énfasis en la actividad mediadora entre documentos y usuarios, los bibliotecólogos nos hemos preparado para trabajar sólo con la cara externa de los conceptos, privilegiando la función comunicativa de las palabras y los símbolos que hacen las veces de fachada de las ideas.

Sin embargo, aun en nuestro propio feudo disciplinario, hemos sido extremadamente descuidados en, por ejemplo, la atribución indiscriminada de etique- 
tas para una sola noción. Así, para representar la idea misma de "concepto", o de "combinación de conceptos", en Organización del Conocimiento, hemos desarrollado la siguiente batería de denominaciones según el contexto de aplicación:

- descriptor (en el ámbito tesaurístico y de otros lenguajes similares),

- encabezamiento de materia o epígrafe (respecto a las listas de encabezamientos),

- palabra clave (para la extracción de conceptos a partir del lenguaje natural),

- foco (para el caso de los lenguajes facetados),

- materia, asunto, tópico o tema (cuando se trata de identificar las temáticas más relevantes y representativas de un documento, y también cuando se hace referencia a los conceptos en los sistemas de clasificación).

El hecho de que hayamos permitido esa dispersión de nombres para designar - más o menos - a lo mismo es, en un ámbito cuya existencia se justifica en gran parte por las tareas de control de vocabulario, un indicador de las dudas y las vacilaciones con que asumimos los aspectos terminológicos, semánticos y epistemológicos de la ingeniería de conceptos.

La preocupación por mejorar nuestro cuerpo de conocimientos y nuestro desempeño acerca del trabajo calificado con conceptos se vuelve acuciante si consideramos el proceso histórico que acompañó a la explosión de la información en el siglo XX en todos sus aspectos (depósito, diversificación de fuentes y de medios de acceso, utilización y reutilización como recurso estratégico, etc.). Ello en gran parte se debe a que los últimos sesenta años han sido testigos, como nunca antes, del surgimiento y el desarrollo vertiginoso de nuevas disciplinas e interdisciplinas, producto de una revolución científico-tecnológica montada en el veloz corcel de los imperativos de las dos guerras mundiales, la posterior guerra fría y los distintos conflictos regionales, así como de la carrera por la conquista del espacio, que tuvo su primera escala tecnológica en la luna.

Naturalmente, las nuevas especialidades y los estudios interdisciplinarios generan literatura. Las disciplinas se construyen mediante delimitaciones temáticas y conceptualizaciones de los objetos de estudio, de las herramientas, los componentes y las aplicaciones de cada nuevo subsegmento del conocimiento. Ello lleva naturalmente a la formulación y estructuración de terminologías que han volcado sobre la sociedad moderna aluviones de nuevos conceptos, muchos de ellos proporcionados por el inglés, lengua instituida como superestrato cultural de nuestros tiempos. Todo ello se expresa en la documentación y obliga a elaborar soluciones metodológicamente aceptables, que estén a la altura de las expectativas y las necesidades de los usuarios de hoy, que están ostensiblemente más

Scire. 6:1 (en.-jun. 2000) 31-53. 
calificados que hace unas décadas para el "autoservicio" en materia de búsqueda y acceso a la información.

En la actualidad parece evidente que los usuarios especializados de información, rodeados y apoyados por un arsenal tecnológico y con la puesta a su disposición de inmensos bancos digitales textuales, referenciales y estadísticos, están preocupados especialmente en identificar cicerones del lenguaje que solucionen sus problemas reales de índole semántica y de vocabulario. Ese nuevo rol mediador cabe de un modo muy natural al bibliotecólogo o documentalista que se especialice tanto en las posibilidades de las nuevas herramientas tecnológicas, como en el estudio de la naturaleza, las características y las leyes de surgimiento, desarrollo y sustitución de lenguaje especializado.

Este artículo pretende argumentar en esa dirección, y proponer el escenario en que ese nuevo profesional de la información deberá situar al tratamiento temático de la información, considerando una perspectiva terminológica.

\section{Terminología y tratamiento temático de la información: esbozo de marco teórico}

La utilidad de la Terminología como disciplina se verifica en tanto ámbito interdisciplinario que se ocupa exhaustiva y exclusivamente de los lenguajes de especialidad, su génesis y desarrollo, sus características e imbricaciones con el quehacer de distintos colectivos profesionales. Hemos escrito ya (Barité, 1999, p. 140) que "la Terminología puede establecerse como un territorio de confluencia de distintas matrices disciplinarias, que se involucran recíprocamente considerando las necesidades de analizar, traducir, formalizar, describir y organizar las voces propias del lenguaje especializado — cualquiera sea el dominio temático a que remita - para fines particulares, no necesariamente intercambiables entre los actores", sean éstos lingüistas, comunicadores, informáticos, traductores o bibliotecólogos.

Conforme con Cabré (1999, p. 100), esta disciplina requiere "aportaciones de tres teorías":

- "una teoría del conocimiento que explique cómo se conceptualiza la realidad, los tipos de conceptualización que pueden darse y la relación de los conceptos entre sí y con sus posibles denominaciones."

- "una teoría de la comunicación", que sé ocupe de la "correlación entre tipo de situación y tipo de comunicación en toda su amplitud y diversidad, y que explique las características, posibilidades y límites de los diferentes sistemas de transmisión de un concepto y de sus unidades".

- "una teoría del lenguaje" que dé cuenta de las unidades terminológicas propiamente dichas dentro del lenguaje natural, y que explique "sus caracte-

Scire. $6: 1$ (en.-jun. 2000) 31-53. 
rísticas gramaticales, semánticas y textuales, [y ] el uso que hacen los especialistas de los términos", entre otras posibilidades

No es concebible pensar que las aplicaciones posibles de la Terminología a la Bibliotecología, en lo que se refiere a reglas y métodos para la organización y la representación de conceptos y de sistemas conceptuales, puedan instrumentarse sin el auxilio de estas tres teorías, pues la mediación entre información y usuario, sea cual sea el contexto en el que se realice, requiere de herramientas hábiles y eficaces de comunicación, bajo determinados acuerdos de lenguaje, y sobre la materia prima del "mensaje", que para nuestro caso es el conocimiento mismo, demandado con una caracterización específica por el usuario.

Entonces, ¿cómo desconocer el impacto que el tratamiento del lenguaje en general y de los lenguajes de especialidad en particular, tiene sobre el análisis y la representación temática de la información, tanto si se opta por trabajar con lenguaje natural, como si se prefiere la elaboración y aplicación de lenguajes controlados? ¿Cómo trabajar en este área dejando a un lado las cuestiones de orden terminológico?

Novellino (1996, p. 38) plantea atinadamente que "la organización del conocimiento en el ámbito de la Ciencia de la Información" no sólo "dice relación con el desarrollo y evaluación de teorías para el análisis de determinadas disciplinas, persiguiendo la elaboración de instrumentos y métodos para la representación de las informaciones generadas en esas disciplinas", sino también, "con la comprensión científica de la estructura del conocimiento, proyectando metodologías para el análisis de conceptos y para la generación de sistemas de clasificación y lenguajes documentales".

Considerando esa doble vertiente, surge naturalmente que sin vocabulario especializado la clasificación, la indización y la organización del conocimiento en general perderían todo sentido u objetivo. Por ello casi podría decirse que la Terminología es, pues, la nutriente natural y esencial para muchas de las actividades especializadas de organización del conocimiento.

Ahora bien, siendo más precisos, ¿cuáles son las líneas que permiten argumentar en favor de la utilidad de la Terminología para la Bibliotecología, en especial en todo lo que hace referencia a la representación y organización del conocimiento mediante conceptos, en tanto unidades cognitivas de mediación entre la documentación y los usuarios de un servicio de información?:

En primera instancia, se trata de una disciplina emparentada con la Lingüística, la Semántica y con la Lexicografía, con las que comparte ciertos marcos teóricos, metodologías y aplicaciones, que han sido tradicionalmente desatendidos por nuestra profesión. Son relativamente recientes los estudios de interfase con estas áreas de conocimiento, y en general han surgido por preocu-

Scire. 6:1 (en.-jun. 2000) 31-53. 
paciones vinculadas a las dificultades para implantar programas de indización automática, o por el análisis y la generación de sistemas expertos obligados a un tratamiento lingüístico de la información.

En segundo término, el aprendizaje sistemático de la disciplina terminológica lleva al fortalecimiento de nuestra formación de base. La precariedad de nuestra formación sobre aspectos y fundamentos lingüísticos ha llevado a que durante muchas décadas hayamos privilegiado -como fue dicho- el trabajo técnico con las etiquetas de los conceptos, su representación verbal o codificada externa, dando por sentadas o sobreentendidas las enunciaciones semánticas que están detrás de esos rótulos. El avance que significó la metodología de construcción de tesauros en cuanto a la formación de estructuras o redes semánticas donde quedan a la vista las relaciones recíprocas entre conceptos fue importante para comprender algunos aspectos formales que subyacen a todo sistema de conceptos. Pero no fue suficiente para consolidar una episteme cuya articulación diera respuesta satisfactoria a muchas polémicas que con un adecuado fundamento se hubieran evitado (como es, el análisis y la valoración confusa y errática que en Teoría de la Clasificación todavía hoy se sigue haciendo respecto de las llamadas relaciones polijerárquicas).

En tercer término, y yendo a expresiones más específicas, la Terminología puede proporcionar soporte teórico, práctico y/o metodológico para resolver distintas situaciones de trabajo profesional relacionadas con el manejo de conceptos. Así:

a) Desde la órbita de los clasificacionistas (o sea, los diseñadores y creadores - o modificadores - de lenguajes documentales), contribuye en tres direcciones no excluyentes:

- Por una parte, permite dominar los aspectos estructurales, los alcances y las fronteras de un dominio temático, el entramado semántico y la red de relaciones que se establecen entre los conceptos propios de ese dominio. Contribuye así, a sistematizar cualquier universo de conceptos, para favorecer el almacenamiento y la recuperación de información sobre bases lógicas y actualizadas.

- En segundo término permite conocer mejor los procesos interiores por los cuales surge nuevo conocimiento y se inserta en las estructuras conceptuales preexistentes en una disciplina.

- En tercer lugar, fortalece los aspectos prescriptivos y normalizadores necesarios en Bibliotecología para la identificación de los términos autorizados y los no autorizados.

- Por último, aporta métodos de análisis terminológico de aplicación a los neologismos en proceso de incorporación a una lengua o a un len-

Scire. 6:1 (en.-jun. 2000) 31-53. 
guaje de especialidad, determinando sus variantes gráficas y sugiriendo criterios sobre la oportunidad y el mérito para su inserción en los lenguajes documentales de uso, mediante parámetros de validación adecuados.

b) Desde la óptica de los clasificadores e indizadores, postula pautas para la ubicación contextual y relativa de los documentos en una colección, atendiendo a sus referenciales temáticos; incorpora nuevos criterios y valoraciones para el análisis de contenido, y propone técnicas de análisis, presentación y contextualización de conceptos tomados por extracción del lenguaje natural.

c) Desde la perspectiva de los referencistas, aporta parámetros para la evaluación terminológica de obras de referencia, e incluso brinda las herramientas teórico-metodológicas para que el bibliotecólogo pueda participar en grupos de trabajo interdisciplinario que se ocupen de la elaboración de diccionarios, glosarios y vocabularios especializados.

Con lo dicho, es posible afirmar que la Terminología ofrece aplicaciones específicas en la búsqueda, la normalización y el acceso a información especializada, y que es capaz de proveer medios de análisis y resolución de problemas tanto en ambientes de lenguaje natural (Internet, bases a texto completo, etc.), como en entornos donde se requiere trabajar con estructuras normalizadas (tesauros, vocabularios, etc.).

Suele suceder que, en algún momento de su vida profesional el bibliotecólogo clasificador o indizador termine lidiando con la necesidad de actualizar, modificar o elaborar vocabularios controlados u otros sistemas de conceptos. Para ello, deberá vestirse con las ropas del clasificacionista, y apelar a métodos de compilación, análisis y validación de términos especializados que no siempre han estado a su alcance en su período de formación. Ese desafío se presenta, más tarde o más temprano, y obliga a considerar la necesidad de una recapacitación específica en Terminología.

Dentro de este marco conceptual bajo el cual analizamos la representación de conocimiento, entendemos de aplicación el modelo llamado "de enfoque pragmático de la información" que postula Rendón Rojas (1996), pues atiende tanto a los aspectos formales como a los semánticos de los conceptos y los modos en los que se expresa el conocimiento, y por ende, la información, que no es otra cosa que "conocimiento registrado" al decir de Guimaraes (1999), o "conocimiento socializado" como hemos afirmado en otra parte (Barité, 1999).

Para Rendón Rojas (1996, p. 27) “el mundo de la información está "habitado“- - usando terminología fregeana- por el sentido de las expresiones, por los contenidos de conciencia y por las intenciones del alma, dirían los fenomenólo-

Scire. 6:1 (en.-jun. 2000) 31-53. 
gos y los medievales; por los significados de los enunciados, por las formas lógicas del pensamiento y las relaciones entre ellas, por los postulados de significación, y por otros objetos."

Conforme con esa demarcación en favor de un enfoque pragmático de la información, concibe ese autor mexicano a ésta "como una cualidad secundaria de los objetos, que es producida por los sujetos a partir de la estructuración de propiedades presentes en esos objetos"... “La información es producto de una síntesis de elementos objetivos y subjetivos, de propiedades reales de los objetos con la actividad del sujeto"..."Como resultado de esa estructuración, — sigue Rendón Rojas—, se construye un ente ideal que se objetiviza y llega a formar el mundo de la información.. Para su elaboración se toma cierta materia prima: elementos objetivos que se encuentran en las cosas...,pero tampoco se descubre como un ente objetivo per se, porque no es algo que existe ya hecho; es necesaria la acción de un sujeto para su aparición. Un documento no tiene información de la misma manera que una bolsa contiene manzanas; el sujeto puede enfrentarse con las manzanas, sólo es necesario que busque en el lugar indicado; pero en el documento no es así, la información surge únicamente cuando el sujeto a través de ese documento llega al mundo de la información; ese paso del símbolo a la información, es una actividad que se realiza y se repite cada vez que se lleva a cabo la estructuración de datos por diferentes sujetos o por el mismo sujeto, pero que cada vez da como resultado más o menos el mismo producto. Si no se conocen las reglas de estructuración para que esos símbolos nos lleven al mundo de la información, el documento puede estar ahí enfrente de nosotros, como los jeroglíficos olmecas, y no decir nada."

\section{La noción de "concepto" o el concepto de "noción"}

Si es cierto, como asegura — contundente— la autoridad de Salvador (1983, p. 51ss.) pese a las diatribas de algunos lingüistas, que "sí hay sinónimos", o sea "palabras que puedan ser utilizadas por su poseedor indistintamente —e independientemente de conveniencias contextuales o de intenciones momentáneasno para designar sino para significar una cosa", es muy probable que el vínculo entre los términos "noción” y "concepto" se encuadre en esa categoría relacional.

La organización y el desarrollo del discurso científico en general, y su estructuración lógica interna, no son concebibles sin la apelación constante a dos elementos que necesariamente sustentan, fundamentan e integran cualquier sistema de ideas: los conceptos y sus definiciones.

La Organización del Conocimiento en su consecuencia última y más simplificada, no es otra cosa que un despliegue articulado y sistemático de conceptos, 
intervinculados en razón de determinada filosofía o concepción epistemológica de partida.

Para la norma internacional ISO 704 (1987, p. 1), destinada a "establecer los principios y los métodos que regirán la unificación y la normalización de la elaboración de las normas terminológicas, tanto en el plano nacional como en el internacional", las nociones son "construcciones mentales que sirven para clasificar los objetos individuales del mundo exterior o interior [...] de una abstracción más o menos arbitraria”.

Boutin-Quesnel et al (1985, p. 18) definen a la noción como "unidad de pensamiento constituida por un conjunto de caracteres atribuidos a un objeto o a una clase de objetos, y que puede manifestarse por medio de un término o un símbolo."

El diccionario TERMCAT de Lingüística (1992, p. 48) dice que es "la representación mental de una clase de objetos que se construyen por abstracción a partir de las propiedades comunes de los objetos individuales que la integran".

Si bien es una representación mental, "un concepto se distingue de una imagen en que ésta posee características concretas comunes con algún objeto determinado, mientras que el concepto sólo contiene características generales, esenciales y definitorias obtenidas por abstracción" (Martínez Riu y Cortés Morató, 1992).

Si intentamos un mínimo acuerdo respecto a los rasgos definitorios de lo que entendemos como noción o concepto, es posible identificar los siguientes:

1) Todo concepto es una construcción abstracta, que utiliza al lenguaje como vía para representar (o sea, volver a presentar) de un modo comprensible o inteligible, un objeto o un fenómeno de la realidad (presente, pasada o incluso futura) o del reino de lo ideal.

2) Todo concepto o noción corresponde a la expresión de una unidad aislable, autónoma de pensamiento. Es, para decirlo de otra manera, una parte significativa de la realidad material o ideal sobre la cual la reflexión humana ejerce una segmentación o delimitación convencional. Como señalan Martínez Riu y Cortés Morató (1992) "según la interpretación tradicional, como representación mental que son, [los conceptos] son necesarios para pensar las cosas, en el sentido de que sólo el concepto posee la suficiente determinación que hace posible el reconocimiento y comprensión de lo percibido por los sentidos".

3) Asimismo, y por consecuencia, cada concepto o noción se constituye en una unidad mínima y autosuficiente de conocimiento. 
4) La construcción de un concepto implica necesariamente su definición, usualmente por medio de claves de lenguaje, como se verá.

5) Desde una óptica terminológica, todavía es posible agregar un quinto rasgo o atributo de las nociones, y es que cada una de ellas, en tanto unidad orgánica de conocimiento, se inserta necesariamente en una rama, una parte, una disciplina en suma, del saber especializado. No cabe hablar de conceptos en Terminología sin el marco referencial de una disciplina (científica, técnica) o de una especialidad, y del sistema de conceptos que la representa. Ausente ese marco, sólo encontramos palabras de la lengua general con sus significados, pero no conceptos especializados, pues los conceptos son en última instancia, unidades cognitivas que adquieren su significación plena sólo en el contexto de un sistema nocional disciplinario.

\section{La construcción de un concepto}

Conviene detenerse a analizar el modo en que se define y se representa una noción o conceptualización, pues tiene implicancias teóricas y metodológicas sobre el tratamiento temático de la información que realiza un bibliotecólogo.

Comencemos por decir que en toda conceptualización podemos identificar dos planos o caras:

1. Una cara interna, que refiere, comprende y abarca al conjunto de enunciados que definen a ese concepto, los que se suceden desde el más general hasta el más específico que sea necesario para identificar al objeto o fenómeno conceptualizado de un modo inequívoco. Los enunciados deben ser breves y precisos. Los hay de cuatro especies:

a) Esenciales, aquellos cuya presencia es absolutamente necesarios para definir inequívocamente al objeto.

b) Accidentales, los que aportan rasgos o atributos que no son generalizables sino a una parte o a ciertos tipos del objeto.

c) Informativos, los que proporcionan datos o elementos que contextualizan o facilitan la comprensión del objeto.

d) Históricos, que favorecen un análisis retrospectivo del objeto y sus mutaciones en el tiempo.

2. Una cara externa, que corresponde a la palabra, el conjunto de palabras u otro tipo de símbolo que identificará y representará al concepto. Esta cara externa o "fachada del concepto", es la que facilita y simplifica los procesos de comunicación entre dos o más personas, pues es lo que se 
utiliza como moneda de intercambio sustituyendo al conjunto de las enunciaciones del concepto, las que se dan por sobreentendidas.

En Clasificación e Indización, la fachada del concepto se expresa, según el tipo de lenguaje documental o la modalidad de indización que se utilice para representar el contenido temático de los documentos, en cuatro tipos de formas:

a) Por medio de una palabra aislada. Esta es la modalidad más aconsejable, porque concentra la conceptualización en un signo.

b) Por medio de un conjunto de palabras o sintagmaA este respecto pueden darse dos posibilidades: una, que la expresión sintagmática manifieste un sólo concepto expresado en más de una palabra, en lo que podría llamarse precoordinación léxica (por ejemplo: fuga de cerebros, cosa juzgada). Otra, que el sintagma manifieste una combinación de dos o más conceptos en dos o más palabras, lo que desde el punto de vista de la Teoría de la Clasificación configura una precoordinación semántica.

c) Por medio de la fraseología, es decir, por una expresión compuesta. Conforme con Corpas (1996, p. 17ss.) las unidades fraseológicas - "son unidades léxicas formadas por más de dos palabras gráficas en su límite inferior, cuyo límite superior se sitúa en el nivel de la oración compuesta". Agrega que "dichas unidades se caracterizan por su alta frecuencia de uso, y de coaparición de sus elementos integrantes; por su institucionalización, entendida en términos de fijación y especialización semántica [y] por su idiomaticidad y variación potenciales". De acuerdo con Corpas, los sintagmas quedan entonces incluidos en la definición de lo que es fraseología. Sin embargo cabe precisar que, aunque todo sintagma es una unidad fraseológica, no toda unidad fraseológica es un sintagma. Quizás el elemento distintivo esté en la identificación del sintagma con lo que Stubelius, citado por Jones (1981, p. 55) denomina "unidad semántica". En efecto, por una parte, si bien una unidad semántica puede estar representada por una palabra sola, no es menos cierto que para que un sintagma esté legitimado en términos bibliotecológicos, es importante que responda a una sola conceptualización, o a una combinación de conceptos lo suficientemente aceptada y garantizada por la documentación. Por otra parte, una unidad fraseológica no sintagmática seguramente incluirá más de una unidad semántica, y hasta puede estar involucrada en un proceso de descomposición de la frase en sintagmas menores o más acotados. Así, la expresión "represión de delitos contra edificios públicos que integran el patrimonio histórico" en tanto pueda adquirir rango de fraseología, a vía de ejemplo, por la repetición de esa configuración en textos legales, seguramente será "desarmada" en tres o cuatro 
sintagmas para fines bibliotecológicos: "delitos" o "represión de delitos", "edificios públicos" y "patrimonio público". Y es que la fraseología no sintagmática es antes que un problema que una contribución para la representación de conocimiento. Podemos encontrar todavía, otro elemento diferenciador entre unidad fraseológica y unidad semántica, que vuelve a la primera inapta para fines bibliotecológicos: la inclusión de verbos (incluso conjugados) o adverbios, y la asimilación de refranes y adagios como fraseología.

d) Por medio de un símbolo codificado, proporcionado por un lenguaje documental para representar una determinada disciplina o clase (por ejemplo, 780 significa Música para el Sistema de Clasificación Decimal Dewey). Este símbolo puede ser ideado por los responsables del lenguaje documental, o puede ser tomado de una nomenclatura o clasificación científica.

En los tesauros y en otros lenguajes documentales que utilizan conceptos para la representación de contenido se prefiere excluir la fraseología, aunque suele considerarse un porcentaje variable, pero relativamente alto, de sintagmas para envasar descriptores y no descriptores. La utilización habitual de sintagmas está dada más por imperativos de la realidad lingüística de los conceptos que por sugerencias de la teoría. Los teóricos de la Clasificación recomiendan restringir al máximo el uso de sintagmas, pero la práctica demuestra que, tanto se utilice lenguaje natural o alguna especie de lenguaje normalizado para la representación del contenido, parece tarea imposible evitar la proliferación de formas sintagmáticas. Con la aspiración, quizás, de convivir de un modo satisfactorio con este hecho consumado, los clasificacionistas suelen aconsejar la formación de expresiones compuestas bajo los siguientes criterios de presentación:

a) Un sustantivo más un adjetivo especificador o modificador (por ejemplo, himno olímpico, pala mecánica o fianza penal).

b) Alternativamente, dos sustantivos enlazados por una preposición o una conjunción (por ejemplo, salto con garrocha, libertad de culto).

Los sintagmas son importantes para la representación del conocimiento con fines documentales porque, en algunos casos, dan un sentido particular a dos palabras que separadas no significarían lo mismo; es lo que sucede, por ejemplo, con el término "giro postal". En otros casos, el sintagma permite desambiguar un término vago o especificarlo, como en "compuestos quimicos". Autores como Currás (1998, p. 84ss.), Gil Urdiciain (1996, p. 188ss.), Cavalcanti (1978) o Austin \& Dale (1984) han estudiado con detalle las maneras más adecuadas de implantar sintagmas en lenguajes documentales. Otman (1996) aporta una visión 
más terminológica pero que tiene también clara aplicación en nuestra área disciplinaria.

Cabe señalar que la utilización a mediana y gran escala de sintagmas obliga, por regla general, a incorporar herramientas adicionales (como ser índices KWIC), para multiplicar los puntos de acceso por palabras aisladas a la documentación de la base.

Con lo dicho, es posible establecer una tríada de elementos que están presentes inevitablemente en un proceso de conceptualización (Fig. 1):

- Un objeto (material o ideal; entidad o fenómeno; existente, existido o presunto).

- Un concepto como manifestación abstracta de ese objeto.

- Una expresión lingüística o simbólica como representación del objeto y de su concepto, para fines de comunicación.

Su expresión gráfica más representativa es la de un triángulo en la medida en que ninguno de los tres vértices subsiste por sí mismo en un escenario humano, pues de lo contrario sería imposible la comunicación de ideas y la formulación de conocimiento. Dicen algunos autores que la Terminología es la ciencia de los términos (y no la ciencia de los conceptos), pero hay en esa divergencia más una cuestión de grado que de esencia.

Y es que cuando en Terminología se hace referencia a los términos, generalmente suelen confundirse dos significaciones, utilizadas en forma indistinta:

a) El término como sinónimo, apenas, de la representación o "etiqueta" de un concepto, esto es, su cara externa: la palabra, la expresión o el símbo-

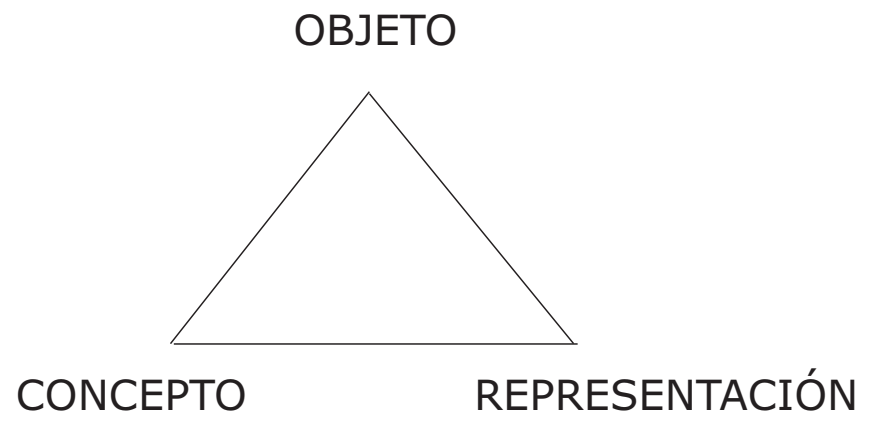

Fig.1. Elementos presentes en un proceso de conceptualización

Scire. $6: 1$ (en.-jun. 2000) 31-53. 
lo que en un determinado lenguaje expresa y representa una noción abstracta. Esta es la acepción más usual de la palabra "término" en la literatura de la disciplina. Así lo entiende Dubuc (1999, p. 55), cuando expresa que todo término, como "elemento constitutivo de cualquier nomenclatura terminológica relacionada con una lengua de especialidad, es la denominación de un objeto propio de una determinada área de especialidad". En este caso, el término es definido por su valor de economía para facilitar y establecer la comunicación de aquello que designa, connota o comprende, pues pasa a constituir el elemento de comprensión recíproca entre dos interlocutores en un determinado nivel de discurso. Es, en suma, en cuanto etiqueta-resumen de un concepto, pieza de intercambio en una comunicación. Si trasladamos esta idea a una especie de fórmula, diremos que "término = representación de un concepto", o sea $(\mathrm{T}=\mathrm{R})$. En esta línea de argumentación, la palabra "Litisconsorcio" sería el término del Derecho Procesal asignado al concepto "Litigio en el que participan de una misma suerte varias personas" (Peniche, 1997, p. $35)$.

b) El término como expresión abarcadora de la noción o conceptualización abstracta de un objeto y de su representación. Si bien esta acepción es menos corriente, enlaza de mejor manera con una perspectiva integral de una noción, que alcance a la idea y a su correlato en el lenguaje. En este caso, la fórmula puede expresarse como término $=$ noción + representación $(\mathrm{T}=\mathrm{N}+\mathrm{R})$. En este sentido Cabré $(1995$, p. 20) señala que "para la filosofía, la terminología es un conjunto de unidades cognitivas que representan el conocimiento especializado". Y que "estas unidades tienen una doble vertiente: por un lado son unidades de conocimiento, por cuanto los hablantes se aproximan al mundo a través de ellas; por otro lado, son unidades de representación, que dan una idea de la organización del mundo especializado". En este caso, el término comprendería, para el ejemplo arriba indicado, tanto a la palabra "Litisconsorcio" como a su definición.

También hay quien ha invertido esta fórmula indicando que "concepto es igual a noción + representación”. Así, Dalhberg (1978, p. 12) ha escrito que "concepto es la unidad de conocimiento que surge por la síntesis de los predicados necesarios relacionados con determinado objeto y que, por medio de signos linguiísticos [los términos para este caso] puede ser comunicado."

\section{La definición de un concepto}

Existen distintas formas de definir un concepto; la más común es la denominada definición por comprensión o intensión (sic). Este tipo de definición se veri-

Scire. 6:1 (en.-jun. 2000) 31-53. 
fica mediante la formalización de enunciados, que se suceden y se complementan, siendo el primero el más general, y los subsiguientes cada vez más específicos a los anteriores. Al decir de Martínez Riu y Cortés Morató (1992), "la comprensión es el conjunto de las características o rasgos definitorios" de algo, los que se acomodan ordenadamente en enunciados que agregan un rasgo o atributo más respecto al anterior. Dubuc $(1999$, p. 58) reafirma esta idea cuando dice que "el concepto es la reunión de los rasgos característicos del objeto designado por el término".

El principio involucrado en este tipo de definición es el de género próximo y diferencia específica, por el cual el primer enunciado corresponderá al género más amplio al que pertenece el objeto, y a partir de allí cada enunciado presentará una diferencia respecto al género más inmediato, lo que irá particularizando cada vez más al objeto analizado, hasta lograr que no se confunda con ningún otro. De este modo, si la metodología enunciativa se aplica correctamente, la definición formada por la sumatoria de los enunciados sólo podrá corresponderse con el objeto, y no involucrar a ningún otro similar o de naturaleza afín pero distinto.

La definición por comprensión o intensión es, en realidad, la más común en el discurso científico o especializado, y en los diccionarios y otras obras de referencia. La Real Academia Española, por ejemplo, organiza las definiciones de la lengua aplicando esta metodología con especial rigor. De todos modos, cabe consignar que un concepto puede de ser definido de muchas maneras, considerando los mismos o distintos enunciados, o haciendo prevalecer -por ejemplo- los enunciados informativos o accidentales sobre los esenciales. La definición de enunciados también varía conforme al destinatario de la misma, y ello genera distintos tipos de discurso: especializado, de divulgación o de vulgarización del concepto.

Tomemos, para ejemplificar el procedimiento de intensión, un caso de diccionario especializado, descomponiendo la definición de los términos en sus enunciados:

COMPETICIÓN (Diccionari General dels esports olímpics, 1992, p. 65).

1. "Manifestación deportiva ..." (primer enunciado esencial que indica el género próximo)

2. “... consistente en el enfrentamiento ..." (segundo enunciado esencial que aplica una diferencia específica, pues no toda manifiestación deportiva es competitiva).

3. “... individual o por equipos, ...." (en este caso se trata de un enunciado que indica rasgos accidentales, puesto que es indiferente a la esencia del concepto que el enfrentamiento sea entre personas o entre equipos)

Scire. 6:1 (en.-jun. 2000) 31-53. 
4. “...de dos o más adversarios...” (tercer enunciado esencial -adversariosprecedido de un segundo enunciado accidental -dos o más-)

5. “...para determinar un ganador.” (cuarto enunciado esencial).

Véase como sólo mediante la acción combinada de los cuatro enunciados esenciales puede definirse el concepto "competición" dentro de la especialidad del Deporte, de un modo indiscutible y satisfactorio, pues en los tres niveles antecedentes el concepto puede confundirse con otros. Así:

- Los ejercicios gimnásticos que una persona realiza en su casa son también una manifestación deportiva.

- Una práctica informal entre dos tenistas, previa a un partido oficial, es también un enfrentamiento.

- Los tenistas que se enfrentan en esa práctica informal son, sin duda, adversarios, pues golpean la pelota hacia sectores opuestos de la cancha.

No obstante, en ninguno de los tres ejemplos provistos podemos hablar fundadamente de "competición", pues falta el cuarto enunciado: la necesidad de determinar un ganador.

De un modo indirecto, la definición por comprensión se verifica en los lenguajes documentales a través de la enumeración de los distintos niveles jerárquicos que preceden a la situación de un término. En este sentido, los lenguajes documentales permiten establecer los géneros próximos en los conceptos de notación o ubicación más genérica, y las diferencias específicas en las notaciones más extensas o las ubicaciones más "profundas" de un tesauro. Así, si desvela-

3 Ciencias Sociales.

34 Derecho

347 Derecho Privado. Derecho Civil.

347.7 Derecho de autor.

347.77 Propiedad industrial, comercial y científica.

347.771 Patentes

Figura 2. Contexto genérico del concepto "patentes" 
mos la cadena de conceptos existente tras la notación del concepto "Patentes" en la Clasificación Decimal Universal (1995), logramos el desarrollo de la figura 2.

Este esquema nos permite decir, al menos, que las patentes son objeto de atención como una manifestación de la Propiedad Industrial, o en sentido más amplio, de los Derechos de Autor, y que, en ese orden, son reguladas por el Derecho en tanto Ciencia Social. Algunos rasgos propios de las patentes podrían extraerse de subdivisiones más específicas de 347.771 si existieran.

\section{La relacion dialéctica entre el concepto y su representación}

El concepto no es un compartimiento estanco, ni un núcleo cerrado o autosuficiente separado de su representación. Ni viceversa. Entre la cara interna y la cara externa de una noción discurre una tensión dialéctica que no suele ser pacífica ni dotada de perennidad. Más bien, el grado de estabilidad o perdurabilidad que logra toda entidad "concepto/representación" está sustentada en materia en permanente movimiento.

Es cierto, y no puede desmentirse, que el concepto suele permanecer siendo el mismo en tanto sus representaciones varían de idioma en idioma. Así, la idea que tenemos de un "agujero negro" no varía porque su etiqueta esté en inglés, en noruego o en español. Pero no es menos cierto que, en muchos casos, las variaciones locales modifican hasta cierto grado la enunciación de los conceptos, y esto puede deberse tanto a razones fácticas (un concepto relativo al petróleo puede modificarse por razones geológicas, por ejemplo, según el país en que se sitúe la explotación petrolífera) como legales (los mismos institutos jurídicos pueden ser definidos de modo distinto en distintos países), o ideológicas (ciertas conceptualizaciones económicas podrán asumir diferentes enunciaciones según la doctrina o la ideología desde la que se parta).

Si bien el conjunto de enunciados que constituyen la médula de una noción son ciertamente estables, algunos de esos enunciados —entonces- pueden variar o ser sustituidos por otros, debido a la evolución tecnológica, la aparición de nuevas teorías o enfoques, o a situaciones de hecho. Así, los enunciados que definían al objeto "televisor" en la década del 50 no son los mismos que pueden utilizarse hoy, en razón de que los dispositivos, las características externas y las aplicaciones de un televisor han variado notablemente en estas décadas, sin dejar de ser esencialmente el mismo aparato.

En la misma línea, la evolución del conocimiento vuelve obsoletos, ya ciertos enunciados de un concepto, ya las palabras utilizadas para representarlos. Este fenómeno es habitual particularmente en las ciencias sociales. Otras veces es el concepto mismo el que desaparece porque ya no cumple ninguna función útil en el discurso y en la práctica humana.

Scire. 6:1 (en.-jun. 2000) 31-53. 
La disciplina semántica tiene mucho que decir todavía respecto a los matices de sentido y las coloraciones que una palabra (y por extensión también un término) puede asumir en distintos contextos comunicativos o en diferentes tipos de discurso. Apunta Bouveret (1998, p. 405) que "la construcción del sentido de un término especializado se realiza en una interacción entre lengua y discurso", y ese es un aserto particularmente atinado respecto al discurso científico que se preocupa por analizar, describir, definir, descomponer y recomponer sus objetos de estudio transformándolos — para su mejor dominio- en nociones conceptuales de alta complejidad abstracta.

La traslación recíproca de sentidos entre las palabras de la lengua general y los términos de los lenguajes de especialidad, considerados como subcódigos de aquella lengua genera una constante movilización de los predicados de un concepto, y ha sido estudiada con especial tino por Paz Battaner (1996). Asimismo, los procedimientos de creación de nuevos términos por analogía, metáfora o extensión de sentido — tan comunes en Terminología — y los problemas que plantean para el control de vocabulario, han sido estudiados recientemente en nuestra área por autores como Hamilton (2000, p. 111 ss.) y Stambuk (1998, p. 373ss.). Por estos mecanismos es que encontramos, y asumimos con naturalidad, el término "virus" en Informática o el término "diagnóstico" en evaluación de organizaciones.

Por otra parte, más allá de la aspiración de univocidad de los términos, que durante décadas han propugnado los terminólogos wüsterianos, la realidad confirma todos los días el reinado de la polisemia incluso en las disciplinas científicas más "duras". Nuestra constante preocupación por precisar el alcance de términos, mediante scope notes o indicaciones entre paréntesis del ámbito de uso de un descriptor convalida esa realidad.

La comparecencia simultánea de varias etiquetas para una sola conceptualización (lo que comunmente llamamos sinonimia), o la sustitución de una noción por otra en tanto la etiqueta o palabra de fachada mantiene su vigencia, son fenómenos que también merecen un estudio autónomo y más particularizado que el presente.

La presentación de terminología bajo apariencias "políticamente correctas" o "políticamente incorrectas" también está empezando a ser estudiada, pues genera - en esa interacción entre lengua y discurso de la que hablaba Bouveretreflexiones que van al núcleo mismo de lo que las palabras quieren - o medianamente pueden - representar. Hace unos meses escuchamos por la radio, en la misma semana y en forma casual, un análisis de un sociólogo muy a la moda sobre lo que entendía por "globalización", luego un discurso del presidente de Brasil, Fernando Henrique Cardozo, en el que se quejaba de las prácticas comer- 
ciales proteccionistas de Estados Unidos y señalaba que más que de globalización debería hablarse de "globalización asimétrica". Y en lo inmediato leímos un recorte de prensa en el que un filósofo mexicano, cuyo nombre no conservamos, afirmaba que lo que el mundo llamaba globalización no era otra cosa que "imperialismo" bajo una nueva etiqueta, y hacía una serie de consideraciones al respecto, en las que trataba de redifinir un término y otro.

Otro nivel de problemas es el que se da entre las equivalencias lingüísticas, es decir, las equivalencias de etiquetas y nociones entre una lengua y otra. Quienes más sufren las discordancias conceptuales entre comunidades lingüísticas con fondos culturales diferentes son los traductores, pero sus ramalazos alcanzan a la Bibliotecología especialmente en el trabajo de referencia, y en la preparación de equivalencias para tesauros, o en la indización de documentos en lengua extranjera.

Los componentes y las repercusiones de este vínculo vivo y movedizo entre concepto y representación siguen siendo estudiados en nuestra disciplina, sobre todo en lo que dice relación con el proceso de selección de términos candidatos a ser descriptores, con el registro de altas y bajas de un lenguaje documental, y con los trabajos de normalización de vocabulario. Los procesos neológicos y los de obsolescencia de conocimiento son el gran telón de fondo sobre el que se despliega esta temática. Si no logramos comprender, desde la Bibliotecología, la naturaleza y las repercusiones de estos fenómenos sobre nuestros trabajos con conceptos, difícilmente podamos contribuir a la construcción de una teoría que tiene albañiles de muchas procedencias: traductores, lingüistas, filósofos de la ciencia, epistemólogos, informáticos y comunicadores.

A modo de ejemplo, ¿cómo podríamos validar los términos candidatos a descriptores (o sea, a representantes de conceptos) si no es mediante la aplicación de principios que, como el de respaldo o la garantía documental, implican el análisis de la documentación de un área de modo tal que refleje la existencia, la representatividad y la adecuación de un término para significar un concepto determinado. Ese análisis valorará tanto aspectos estadísticos (la ocurrencia o coocurrencia de términos), como aspectos lingüísticos (la composición morfosintáctica preferente) o terminológicos (la conceptualización y la ubicación en un sistema de conceptos o árbol de dominio del término), entre otros.

\section{Conclusiones}

Las metodologías desarrolladas por la Terminología y la Teoría de los Conceptos pueden, con las debidas adecuaciones, ser utilizadas con provecho en nuestro ámbito para las finalidades expresadas, en razón de que procuran una presentación unívoca de términos (es decir, por cada concepto un término), la nor-

Scire. 6:1 (en.-jun. 2000) 31-53. 
malización en su presentación gráfica y sintáctica, la desambiguación mediante el control de la polisemia y la homonimia, y el adecuado control de equivalencias semánticas y lingüísticas entre los conceptos en distintos idiomas.

Ha pasado ya, afortunadamente, el tiempo en que las experimentaciones informáticas con conceptos, al decir de Moreiro y Méndez (1999, p. 16), llevaban a considerar "las palabras como objetos". Los términos son sí, conceptualizaciones objetivadas, pero nunca objetos, sino la expresión de artefactos abstractos de extrema complejidad, que exigen una aproximación multidisciplinaria para describirlos y utilizarlos con excelencia para nuestras finalidades.

Para alcanzar esa excelencia teórico-metodológica es que propugnamos la necesidad de incorporar asignaturas, ya sea a nivel de grado o de posgrado, que provean una formación específica en Terminología, y en un rosario de asignaturas afines desde la óptica de nuestras prácticas significativas (Semiótica, Lexicografía, Semántica), así como una preocupación mayor por analizar y describir las implicaciones de la implantación de las nuevas herramientas y posibilidades tecnológicas al tratamiento de conceptos para finalidades de almacenamiento y recuperación de información.

Ese terreno en el que tenemos una formación de base particularmente apta para trabajar interdisciplinariamente con lingüistas e informáticos, si hasta hoy ha sido escasamente explorado por nosotros debido a las fragilidades en nuestra formación, abre por su vez insospechadas perspectivas para el tratamiento temático de la información.

\section{Referencias}

Austin, Derek ; Dale, Peter (1984). Directrices para el establecimiento y desarrollo de tesauros monolingües. $2^{\text {a }}$. ed. revisada. París : Unesco, PGI y Unisist, 1984. 86 p.

Barité, Mario (2000). Diccionario sobre Organización y Representación del Conocimiento : Clasificación, Indización, Terminología. URL $=\langle$ www.eubca.edu.uy/diccionario $>$

Barité, Mario ; Guimaraes, José Augusto (1999). Guía metodológica para el acceso, el análisis y la organización temática de documentos jurídicos. Montevideo : Universidad de la República, CCEP, 1999. 161 p.

Barité, Mario (1999). Organización del Conocimiento: un nuevo marco teórico-conceptual en Bibliotecología y Documentación. Ponencia presentada ante el III Simpósio em Filosofia e Ciência, Universidade Estadual Paulista, Campus de Marília, 7 al 10 de junio de 1999. Inédito.

Boutin-Quesnel, Rachel ; [et al] (1985). Vocabulaire systématique de la terminologie. Quebec: OLF, 1985.

Bouveret, Myriam (1998). Approche de la dénomination en langue spécialisée. // Meta. 43: 3 (1998) 393-410.

Scire. 6:1 (en.-jun. 2000) 31-53. 
Cabré, María Teresa (1995). La Terminología: representación y comunicación. Barcelona : IULA, Universitat Pompeu Fabra, 1999. 369 p.

Cavalcanti, Cordelia (1978). Indexaçao \& Tesauro: metodologia \& técnicas. Ed. preliminar. Brasilia : ABDF, 1978. 89 p.

Cintra, Anna Maria Marques; et al (1994). Para entender as linguagens documentárias. Sao Paulo: Polis; APB, 1994. 72 p.

Clasificación Decimal Universal : CDU (1987). Edición abreviada española. $5^{\text {a }}$ ed. revisada y actualizada. Madrid : AENOR, 1987. 358 p.

Corpas Pastor, Gloria (1996). Manual de fraseología española. Madrid : Gredós, 1996.

Currás, Emilia (1998). Tesauros: manual de construcción y uso. Madrid, 1998

Dahlberg, Ingetraut (1978). Fundamentos teórico-conceituais da classificaçao. EN Revista Biblioteconomia Brasilia. 6 : 1 (jan.-jun. 1978) 9-21.

Dubuc, Robert (1999). Manual práctico de Terminología. $3^{a}$ ed. corregida y actualizada. Santiago de Chile : Unión Latina ; Pontificia Universidad Católica de Chile, 1999.

Gil Urdiaciaín, Blanca (1996). Manual de lenguajes documentales. Madrid : Noesis, 1996

Guimaraes, José Augusto Chaves (1999). Palestra presentada ante el III Simpósio de Filosofia e Ciência, Universidade Estadual Paulista, Campus de Marília, 7 al 10 de junio de 1999. Inédito.

Hamilton, Anne (2000). Interface metaphors and logical analogues: a question of Terminology. // Journal of American Society for Information Science. 51 : 2 (2000) 111-122.

Jones, Kevin (1981). Problems associated with the use of compound words in thesauri, with special reference to BS 5723:1979. // Journal of Documentation. 37 : 2 (june, 1981) 53-68.

International Organization for Standardization (1987). Norme internationale ISO 704. París : 1987.

Martínez Riu, Antoni ; Cortés Morató, Jordi (1992). Diccionario de filosofía Herder. Ed. en CD-ROM. Barcelona : Hender, 1992.

Novellino, María Salet Ferreira (1996). Instrumentos e metodologias de representaçao da informaçao. // Informaçao\&Informaçao, Londrina, 1 : 2 (jul.-dez. 1996) 37-45.

Otman, Gabriel (1996). Les représentations sémantiques en Terminologie. París : Masson, 1996.

Paz Battaner, María (1996). Terminología y diccionarios. // Jornada Panllatina de Terminologia. Barcelona : IULA, Universitat Pompeu Fabra, 1996.

Peniche, Surya (1996). Terminología del Derecho Penal. México : UNAM; CUIB, 1997. $106 \mathrm{p}$.

Rendón Rojas, Miguel Ángel (1996). Hacia un nuevo paradigma en Bibliotecología. // Transinformaçao, $8: 3$ (set./dez. 1996) 17-31.

Salvador, Gregorio (1983). Semántica y lexicología del español: estudios y lecciones. Madrid : Paraninfo, 1985.

Stambuk, Anuska (1998). Metaphor in scientific communication. // Meta. 43 : 3 (1998) 373-379. 
Los conceptos y su representación: una perspectiva terminológica ...

TERMCAT (1992). Diccionari de lingüistica. Barcelona : Fundació Barcelona, 1993.

Scire. $6: 1$ (en.-jun. 2000) 31-53. 